\title{
BMJ Open Improving prescribing practices with rapid diagnostic tests (RDTs): synthesis of 10 studies to explore reasons for variation in malaria RDT uptake and adherence
}

Helen E D Burchett, ${ }^{1}$ Baptiste Leurent, ${ }^{2}$ Frank Baiden, ${ }^{3}$ Kimberly Baltzell, ${ }^{4}$ Anders Björkman, ${ }^{5}$ Katia Bruxvoort, ${ }^{1}$ Siân Clarke, ${ }^{6}$ Deborah DiLiberto, ${ }^{7}$ Kristina Elfving, ${ }^{8,9,10}$ Catherine Goodman, ${ }^{1}$ Heidi Hopkins, ${ }^{6}$ Sham Lal, ${ }^{6}$ Marco Liverani, ${ }^{1}$ Pascal Magnussen, ${ }^{11}$ Andreas Mårtensson, ${ }^{12}$ Wilfred Mbacham, ${ }^{13}$ Anthony Mbonye, ${ }^{14}$ Obinna Onwujekwe, ${ }^{15}$ Denise Roth Allen, ${ }^{16}$ Delér Shakely, ${ }^{5,17}$ Sarah Staedke, ${ }^{7}$ Lasse S Vestergaard, ${ }^{18,19}$ Christopher J M Whitty, ${ }^{7}$ Virginia Wiseman, ${ }^{1,20}$ Clare I R Chandler ${ }^{1}$

To cite: Burchett HED, Leurent B, Baiden F, et al. Improving prescribing practices with rapid diagnostic tests (RDTs): synthesis of 10 studies to explore reasons for variation in malaria RDT uptake and adherence. BMJ Open 2017;7:e012973. doi:10.1136/bmjopen-2016012973

- Prepublication history and additional material is available. To view please visit the journal (http://dx.doi.org/ 10.1136/bmjopen-2016012973).

Received 7 June 2016 Revised 22 October 2016 Accepted 17 November 2016

CrossMark

For numbered affiliations see end of article.

Correspondence to Helen E D Burchett; helen. burchett@Ishtm.ac.uk

\section{ABSTRACT}

Objectives: The overuse of antimalarial drugs is widespread. Effective methods to improve prescribing practice remain unclear. We evaluated the impact of 10 interventions that introduced rapid diagnostic tests for malaria (mRDTs) on the use of tests and adherence to results in different contexts.

Design: A comparative case study approach, analysing variation in outcomes across different settings.

Setting: Studies from the ACT Consortium evaluating mRDTs with a range of supporting interventions in 6 malaria endemic countries. Providers were governmental or non-governmental healthcare workers, private retail sector workers or community volunteers. Each study arm in a distinct setting was considered a case.

Participants: 28 cases from 10 studies were included, representing 148461 patients seeking care for suspected malaria.

Interventions: The interventions included different mRDT training packages, supervision, supplies and community sensitisation.

Outcome measures: Analysis explored variation in: (1) uptake of mRDTs (\% febrile patients tested); (2) provider adherence to positive mRDTs (\% Plasmodium falciparum positive prescribed/given Artemisinin Combination Treatment); (3) provider adherence to negative mRDTs (\% P. falciparum negative not prescribed/given antimalarial).

Results: Outcomes varied widely across cases: $12-100 \%$ mRDT uptake; $44-98 \%$ adherence to positive mRDTs; $27-100 \%$ adherence to negative mRDTs. Providers appeared more motivated to perform well when mRDTs and intervention characteristics fitted with their own priorities. Goodness of fit of mRDTs with existing consultation and diagnostic practices appeared crucial to maximising the impact of mRDTs on care, as did prior familiarity with malaria testing; adequate human
Strengths and limitations of this study

- This analysis addresses the gap in knowledge around how to change prescribing practices, a key question in the era of resistance to antimicrobial medicines.

- The analysis exploits indepth data from 10 intervention studies connected through the ACT Consortium in order to explore the reasons for variation in trial outcomes.

- A comparative case study approach was used, allowing trends and patterns to be explored across contexts in a way not possible within single studies.

- By analysing studies conducted within a consortium, access to unpublished documents, raw data and qualitative insights from the study teams allowed a deeper understanding of the studies and their contexts than is often found in systematic reviews of published reports.

- The extent of variation across the study arms in terms of context, provider type, intervention content and study design allowed for exploration of a range of factors affecting outcomes, but also created challenges for comparability, necessitating a case study approach.

resources and supplies; possible alternative treatments for mRDT-negative patients; a more directive intervention approach and local preferences for ACTs.

Conclusions: Basic training and resources are essential but insufficient to maximise the potential of mRDTs in many contexts. Programme design should respond to assessments of provider priorities, expectations and capacities. As mRDTs become established, the intensity of supporting interventions required seems likely to reduce. 


\section{BACKGROUND}

The substantial overdiagnosis of malaria as a cause of acute febrile illness has been the focus of global attention in recent years, ${ }^{1-3}$ given concerns about the clinical effects of misdiagnoses, the cost of first-line artemisininbased combination therapies (ACTs) and emerging malaria drug resistance. ${ }^{45}$ A policy of universal parasitological testing for malaria was introduced by the WHO in $2010,{ }^{6}$ aiming to reduce overprescription of ACTs. ${ }^{2}$ Malaria rapid diagnostic tests (mRDTs) have been developed for use in low-resource settings, making parasitebased testing possible where microscopy may not be available or feasible. ${ }^{4}$

RDTs have been introduced with providers in a range of sectors. ${ }^{7}$ However, evidence from evaluations of mRDT introductions show mixed effects; mRDTs do not lead to improved targeting of ACTs if providers do not consistently use the tests or if they ignore test results. ${ }^{8-12}$ To maximise their potential for improving prescribing practices, evidence is required of the relative success and challenges of different types of mRDT intervention in different contexts.

This paper presents an analysis of the findings from 10 mRDT intervention studies conducted in Africa and Afghanistan, for which indepth information was available about interventions, outcomes and contexts. The studies, all from the ACT Consortium, represent a large proportion of the intervention studies on mRDTs recently conducted in areas of ongoing malaria transmission. This analysis aimed to identify how mRDTs can be used to improve prescribing in different contexts by exploring factors influencing providers' use of and adherence to test results and comparing results of interventions in different settings.

\section{METHODS}

The ACT Consortium is an international research collaboration involving more than 20 institutions working on a systematic series of 25 studies in 10 countries in Africa and Asia, addressing practical questions in the delivery of malaria treatment. ${ }^{13}$ Intervention studies involving mRDTs were conducted in 10 sites in 6 countries. The analysis in this paper focuses on these studies because of the ability it gives to use raw outcome data (allowing comparable outcomes to be calculated), raw data from linked qualitative research, unpublished documentation about intervention content, implementation and contextual information as well as insights from the study teams. This allowed a more detailed and comparable analysis than could be achieved through reliance on publications or quantitative data alone.

This analysis used a comparative case study approach, where each study arm conducted in a distinct setting was considered a case and outcomes were interpreted in terms of the study design, intervention content, implementation and contextual factors. ${ }^{14}$ This approach suits investigation of 'how' and 'why' interventions have an effect and can highlight comparative general trends and distinct patterns that are not visible in single cases. ${ }^{15} 17$ The analysis explored three outcomes:

1. Provider uptake of mRDTs.

The proportion of patients presenting with fever, or history of fever in past 48 hours (unless specified otherwise), who were tested for malaria with an mRDT, as reported by the provider or patient.

2. Provider adherence to positive mRDT results.

The proportion of patients with a positive mRDT result (for Plasmodium falciparum malaria), who were prescribed or received an ACT, the first-line drug for non-severe malaria in all cases, as reported by provider or patient.

3. Provider adherence to negative mRDT results.

The proportion of patients with a negative mRDT result who were not prescribed, or did not receive, any antimalarial as reported by provider or patient (the effect of negative mRDT results on the use of other treatments, including antibiotics, in ACT Consortium studies has been presented in a separate paper). ${ }^{16}$

The analysis evaluated the impact of different interventions to introduce mRDTs in different contexts. Twenty-eight cases (ie, distinct settings or intervention arms) from the 10 studies were included, with a total of 148461 patients (see table 1). Twenty cases from 7 studies analysed mRDT uptake, 24 cases from 9 studies evaluated provider adherence to positive mRDT results and all 28 cases analysed provider adherence to negative mRDT results.

The studies took place between 2007 and 2012. Studies were either individual $(\mathrm{n}=2)$ or clusterrandomised controlled trials $(n=6)$; observational $(n=2)$ or preintervention/postintervention studies $(\mathrm{n}=1)$ (Tanz2 used different designs in their pilot and main study, so $\mathrm{n}=11$ ). Providers targeted were governmental or non-governmental healthcare workers, private retail sector workers or community health volunteers. Six studies took place in East Africa, three in West

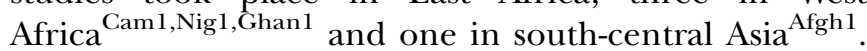
One focused only on children under 5 years ${ }^{\mathrm{Uga} 2}$; the rest included children and adults. See online supplementary file 1 for more detailed information about each study.

All the interventions included basic training on malaria testing with RDTs for healthcare providers, however the content, duration and approach varied. Some interventions included additional activities and materials such as extra training, supervision and feedback, patient information leaflets or school-based activities (see table 2 and online supplementary file 1).

Three studies compared different training packages $^{\text {Nig1,Cam1,Tanz2 }}$. Six studies compared intervention effects in different epidemiological contexts ${ }^{\text {Uga2,Tanz1,Nig1,Cam1,Afgh1,Ghan1 }}$. Seven studies evaluated an intervention against a control arm where mRDTs were not made available Uga1,Uga2,Uga3,Nig1,Cam1,Afgh1, Ghan1. 


\section{Study Study name}

\section{Country Providers targeted}

Afgh1 Strategies for expanding access to quality malaria diagnosis in south-central Asia where malaria incidence is low

Afghanistan Government primary care providers

Cam1 Cost-effectiveness of interventions to support Cameroon the introduction of malaria rapid diagnostic tests in Cameroon

Ghan1 How the use of rapid diagnostic tests influences clinicians' decision to prescribe ACTs

Nig1 Costs and effects of strategies to improve malaria diagnosis and treatment in Nigeria

IMPACT 2: Evaluating policies in Tanzania to improve malaria diagnosis and treatment

Tanz2 Targeting ACT drugs: the TACT trial
Nigeria

Government and mission providers (in hospitals and primary care)

Ghana Government primary care providers

Government and private primary care providers

Government primary care providers, private pharmacies and private medicine dealers

Tanzania

Government healthcare providers (in hospitals and primary care)

Tanzania
Cases $^{*}$

Afgh1/a: training; patients individually

results randomised to receive either $\mathrm{mRDT}$ or established microscopy, Eastern province Afgh $1 / \mathrm{b}$ : training; patients individually randomised to receive either $\mathrm{mRDT}$ or recently introduced microscopy, Northern province

Afgh1/c: training; patients individually randomised to receive either mRDT or clinical diagnosis (no microscopy available), Northern province

Cam1/a1: basic training, Bamenda

Cam1/b1: basic training, Yaoundé

Cam1/a2: enhanced training, Bamenda

Cam1/b2: enhanced training, Yaoundé

Ghan1/a: training; patients individually

randomised to receive either mRDT or microscopy

Ghan 1/b: training; patients individually

randomised to receive either $\mathrm{mRDT}$ or clinical diagnosis

Nig1/a1: basic training, Enugu

Nig1/b1: basic training, Udi

Nig1/a2: enhanced training, Enugu

Nig1/b2: enhanced training, Ud

Nig1/a3: enhanced training + school activities,

Enugu

Nig1/b3: enhanced training + school activities, Udi

Tanz1/a: standard MoH† training, Mwanza,

moderate transmission

Tanz1/b: standard MoH training, Mbeya, low transmission

Tanz1/c: standard MoH training, Mtwara,

moderate transmission

Tanz2/a1: pilot study, low transmission

Tanz2/b1: pilot study, moderate transmission

Tanz2/2: basic training

Tanz2/3: enhanced training

Tanz2/4: enhanced training + patient sensitisation 


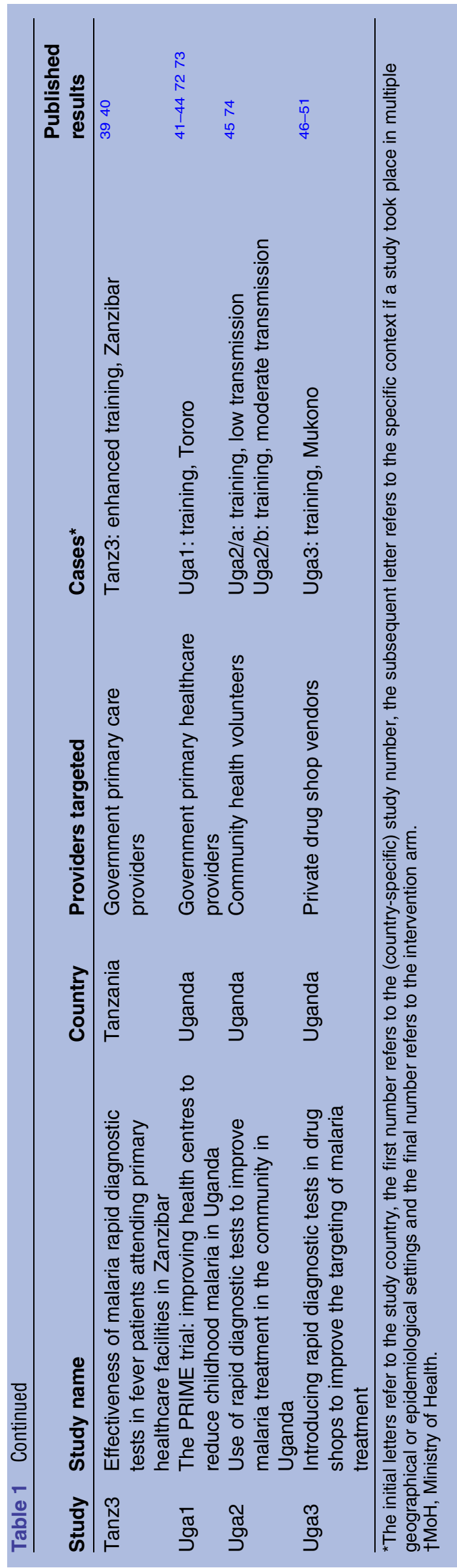

Comparability of findings

Although the studies were co-designed and largely similar, because of differences in primary study questions and differences in epidemiology, data collection methods and evaluation timing, mean pooled analyses would be inappropriate. For example, mRDT uptake was reported through provider-completed registers in some projects and patient exit interviews in others. Some studies reported adherence in terms of the percentage of patients prescribed ACTs or antimalarials, while others reported the percentage of patients who received them. Stockouts may have affected receipt of medication; whether prescriptions were affected is unknown, as alternative medication may or may not have been offered when there was a known stockout. The analysis presented therefore focuses on understanding the reasons for variation in the results, rather than seeking pooled point estimates.

Quantitative outcome data were extracted from each study's raw data set and reanalysed to maximise comparability across studies, using the most comparable denominators and numerators possible. Study, intervention and context characteristics were extracted from published and unpublished documents. Where available, thematic content analysis was undertaken on qualitative data from providers involved in the studies (ie, focus group discussions ${ }^{\text {Uga2,Uga3 }}$ or interviews Afgh1,Ghan1,Tanz1/a,Tanz1/b,Tanz2,Uga1 with health workers, drug shop vendors or volunteers). In Tanz3, interviews from a later, related study were analysed, which included six study providers and six similar providers who had not been involved in the study but had comparable mRDT experiences.

The analysis drew on the approaches informing intervention component analysis (ICA) ${ }^{52}$ and qualitative comparative analysis (QCA), ${ }^{53}$ which seek to identify critical features of interventions. As with ICA, we sought to identify how interventions differed from one another and then, as with QCA, identify which factors appeared to be important. Our initial stage involved gathering as much information about the interventions as possible, going broader than the ICA approach by also capturing information about their delivery and context. However, our analysis differed from ICA and QCA, which attempt to characterise and apply scores to interventions and their characteristics and cross-tabulate these with outcomes. We found our data were not amenable to scoring in a quantitative sense, due to wide variation in the extent and types of information available. Therefore, our analysis was qualitative, using a meaning-based approach. Tables were created for each outcome of interest, with explanatory factors relating to the intervention, context and study design (see online supplementary file 2 for an example). These were shared with study teams and the ACT Consortium core scientific team, with ongoing discussions about the findings and other potential explanatory factors. 
Table 2 Intervention content

\begin{tabular}{|c|c|c|c|c|}
\hline Scenario & mRDT/malaria training & Supervision & mRDT/ACT supplies & Other intervention activities \\
\hline $\begin{array}{l}\text { Afgh1/a } \\
\text { Afgh1/b } \\
\text { Afgh1/c }\end{array}$ & $\begin{array}{l}\text { One and a half day training, following the national training } \\
\text { package. This covered performing mRDTs (most, but not all, } \\
\text { practiced testing) and prescribing antimalarials }\end{array}$ & None & mRDTs supplied by study & None \\
\hline $\begin{array}{l}\text { Cam1/a1 } \\
\text { Cam1/b1 }\end{array}$ & $\begin{array}{l}\text { One day, didactic session covered three modules: malaria } \\
\text { diagnosis, mRDTs, and malaria treatment }\end{array}$ & Monthly & $\begin{array}{l}\text { mRDTs and ACTs supplied } \\
\text { by study }\end{array}$ & None \\
\hline $\begin{array}{l}\text { Cam1/a2 } \\
\text { Cam1/b2 }\end{array}$ & $\begin{array}{l}\text { Same as Cam1/1, plus: } \\
\text { Interactive two day training on adapting to change (focused } \\
\text { on WHO malaria treatment guidelines), professionalism and } \\
\text { effective communication }\end{array}$ & Monthly & $\begin{array}{l}\text { mRDTs and ACTs supplied } \\
\text { by study }\end{array}$ & None \\
\hline $\begin{array}{l}\text { Ghan } 1 / a \\
\text { Ghan1/b }\end{array}$ & $\begin{array}{l}\text { Two day training about the sensitivity and specificity of } \\
\text { mRDTs, alternative causes of febrile illness and the Ghana } \\
\text { national guidelines (which indicated presumptive treatment for } \\
\text { children who are }<5 \text { years old) }\end{array}$ & $\begin{array}{l}\text { None, but study team } \\
\text { were present }\end{array}$ & mRDTs supplied by study & None \\
\hline $\begin{array}{l}\text { Nig1/a1 } \\
\text { Nig1/b1 }\end{array}$ & $\begin{array}{l}\text { Half day demonstration on how to use mRDTs, which } \\
\text { included practising conducting one test. They also received a } \\
\text { copy of the WHO job aid, which shows the steps in using an } \\
\text { mRDT }\end{array}$ & None & mRDTs supplied by study & None \\
\hline $\begin{array}{l}\mathrm{Nig} 1 / \mathrm{a} 2 \\
\mathrm{Nig} 1 / \mathrm{b} 2\end{array}$ & $\begin{array}{l}\text { Same as Nig1/1, plus: } \\
\text { Two day interactive, seminar-style training, covering how to } \\
\text { test, appropriate treatment for positive and negative results } \\
\text { and effective communication. Those attending were given job } \\
\text { aids (eg, treatment algorithm) }\end{array}$ & Monthly & mRDTs supplied by study & None \\
\hline $\begin{array}{l}\mathrm{Nig} 1 / \mathrm{a} 3 \\
\mathrm{Nig} 1 / \mathrm{b} 3\end{array}$ & Same as Nig1/2 & Monthly & mRDTs supplied by study & School-based activities \\
\hline $\begin{array}{l}\text { Tanz1/a } \\
\text { Tanz1/b } \\
\text { Tanz1/c }\end{array}$ & $\begin{array}{l}\text { Two day training (standard MoH), covering performing } \\
\text { mRDTs (including practical) and prescribing antimalarials }\end{array}$ & $\begin{array}{l}\text { Routine } \mathrm{MoH} \\
\text { supervision only }\end{array}$ & mRDTs supplied by $\mathrm{MoH}$ & None \\
\hline $\begin{array}{l}\text { Tanz2/a1 } \\
\text { Tanz2/b1 }\end{array}$ & $\begin{array}{l}\text { One day training on how to use the } \mathrm{mRDT} \text { and read the } \\
\text { result. Antimalarial drug use guidelines were reviewed and job } \\
\text { aids provided }\end{array}$ & None & mRDTs supplied by study & None \\
\hline Tanz2/2 & $\begin{array}{l}\text { Two day, didactic, MoH training on how to use mRDTs, } \\
\text { including practical }\end{array}$ & $\begin{array}{l}\text { Six-weekly, focused on } \\
\text { supplies and reporting }\end{array}$ & mRDTs supplied by study & None \\
\hline
\end{tabular}




\begin{tabular}{|c|c|c|c|c|}
\hline Scenario & mRDT/malaria training & Supervision & mRDT/ACT supplies & Other intervention activities \\
\hline Tanz2/3 & $\begin{array}{l}\text { Same as Tanz2/2, plus: } \\
\text { Three additional } 90 \text { min interactive training workshops, with } \\
\text { one session repeated } 6-7 \text { months later. These covered: } \\
\text { adapting to the change in the diagnosis and management of } \\
\text { malaria; practice with confidence when using mRDTs: tools to } \\
\text { enable change in managing febrile illness; sustaining the } \\
\text { change in practice. Training on communication skills was } \\
\text { included }\end{array}$ & $\begin{array}{l}\text { Six-weekly, focused on } \\
\text { supplies and reporting }\end{array}$ & mRDTs supplied by study & $\begin{array}{l}\text { SMS feedback on own mRDT } \\
\text { uptake and adherence at } 5 \text { months } \\
\text { Two times per day motivational } \\
\text { SMS for } 15 \text { days }\end{array}$ \\
\hline Tanz2/4 & Same as Tanz2/3 & $\begin{array}{l}\text { Six-weekly, focused on } \\
\text { supplies and reporting }\end{array}$ & mRDTs supplied by study & $\begin{array}{l}\text { SMS feedback on own mRDT } \\
\text { uptake and adherence at } 5 \text { months } \\
\text { Two times per day motivational } \\
\text { SMS for } 15 \text { days. Patient leaflets } \\
\text { and posters }\end{array}$ \\
\hline Tanz3 & $\begin{array}{l}\text { Six to } 11 \text { days IMCI training (depending on whether refresher } \\
\text { training or for new health workers) which included malaria } \\
\text { diagnosis and treatment, plus } 1 \text {-week study-specific training } \\
\text { (including good clinical practice, provision of informed } \\
\text { consent, performance and interpretation of mRDT according } \\
\text { to the manufacturer's instructions). One day of the IMCI } \\
\text { training focused specifically on malaria. Training covered } \\
\text { communication skills }\end{array}$ & None & $\begin{array}{l}\text { mRDTs and ACTs supplied } \\
\text { by MoH, with study back up } \\
\text { in the case of stockouts }\end{array}$ & $\begin{array}{l}\text { IMCI training, additional study } \\
\text { salary for providers }\end{array}$ \\
\hline Uga1 & $\begin{array}{l}\text { Two day training session followed a week later by on-site } \\
\text { training in facilities. Training was interactive and included } \\
\text { performing and reading an mRDT, management of a patient } \\
\text { with fever and either a positive or negative mRDT as well as } \\
\text { patient communication. All health workers were invited to } \\
\text { attend the training }\end{array}$ & $\begin{array}{l}\text { Supervision at } 6 \text { weeks } \\
\text { and } 6 \text { months }\end{array}$ & $\begin{array}{l}\text { mRDTs supplied by } \mathrm{MoH} \\
\text { with study back up in the } \\
\text { case of stockouts }\end{array}$ & $\begin{array}{l}\text { Training on patient-centred } \\
\text { services; training in-charges in } \\
\text { health centre management }\end{array}$ \\
\hline $\begin{array}{l}\text { Uga2/a } \\
\text { Uga2/b }\end{array}$ & $\begin{array}{l}\text { Four day interactive training, covering performing and reading } \\
\text { an mRDT, how to prescribe antimalarials, how to deal with } \\
\text { negative cases and communication skills. Providers were also } \\
\text { given pictorial job aids }\end{array}$ & $\begin{array}{l}\text { Close supervision for } \\
\text { first } 6 \text { months (prior to } \\
\text { evaluation) }\end{array}$ & $\begin{array}{l}\text { mRDTs and ACTs supplied } \\
\text { by study }\end{array}$ & Community sensitisation \\
\hline Uga3 & $\begin{array}{l}\text { Four day interactive training to all drug shop vendors, which } \\
\text { covered performing and reading mRDTs, prescribing } \\
\text { antimalarials, how to deal with mRDT negatives and } \\
\text { communicating and negotiating with patients }\end{array}$ & $\begin{array}{l}\text { Close supervision for } \\
\text { first } 2 \text { months (prior to } \\
\text { evaluation) }\end{array}$ & $\begin{array}{l}\text { mRDTs and ACTs supplied } \\
\text { by study }\end{array}$ & Community sensitisation \\
\hline
\end{tabular}

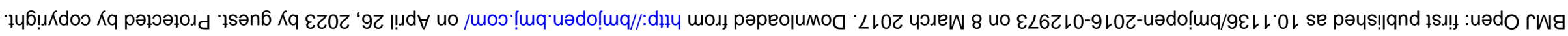




\section{RESULTS}

There was wide variation across cases in all three outcomes: $12-100 \%$ mRDT uptake (figure 1A); 44-98\% adherence to positive mRDTs (figure 1B); $27-100 \%$ adherence to negative mRDTs (figure 1C). All outcomes were universally high in some cases ${ }_{\text {Uga1,Uga2/b,Uga3 }}$ and universally low in others ${ }^{\mathrm{Nig} 1 / \mathrm{a} 1 \text {,Nig1/a3 }}$, but in many cases, the three outcomes did not correspond-for example, testing was infrequent but adherence to results high $^{\text {Tanz1/a,Tanz1/b,Tanz2/3 }}$ or adherence to positives high, but negatives low ${ }^{\text {Ghan1/a,Ghan1/b,Cam1/al,Cam1/b1 }}$, or vice versa $^{\mathrm{Uga} 2 / \mathrm{a}, \mathrm{Nig} 1 / \mathrm{b} 3}$.

There were no single factors which alone accounted for any of the outcomes; successful mRDT uptake and adherence appeared to result from a combination of context and intervention characteristics. The analysis identified several factors which, taken together, may account for the heterogeneity observed. The appeal of the intervention to providers was crucial for all three outcomes, but each was additionally shaped by other factors.

\section{Factors affecting mRDT uptake}

There was wide variation between cases in the use of mRDTs for febrile patients (see figure 1A). Providers' motivation to perform well in the intervention was associated with uptake, as were familiarity with testing, adequate human resources and supplies, and the cost of mRDTs.

\section{Motivation to perform well in the intervention}

The range of sectors and contexts in which providers worked meant that their own priorities varied between cases. For example, government health workers' priorities may have included some or all of the following: treating ill patients, managing their workload in the light of staff shortages, managing (or 'rationing') their medicine supplies in the face of future shortages, maintaining their position of authority as a clinician. In contrast, while private providers may also have prioritised treating ill patients, some viewed their role as more of a business than a healthcare service. As such, their priorities may have been more business-oriented, such as making a profit and ensuring sufficient customers.

Data on provider priorities were not available for all cases; for some, qualitative data were available but for others, anecdotal evidence and study team perceptions were used. Nevertheless, where the intended use of mRDTs and associated intervention activities aligned well with providers' own priorities, they appeared more motivated to participate and 'perform' well in the intervention, and we observed higher uptake and adherence. There were a number of explanations for, and/or factors associated with, higher motivation but political and financial support were often critical. For example, in Tanz2, carefully developed messages addressing existing provider principles and practices, as well as Ministry of Health branding of the intervention (an institution known to influence the government health workers in this setting), appeared to motivate providers. In Uga3, the drug shop vendors were previously not permitted to offer testing and this new service, along with the associated training, supervision and visible involvement of the Ministry of Health, gave them a legitimacy they had previously lacked. ${ }^{48}$ These vendors also reported increased customer numbers and associated profits, enhanced by the study's free provision of mRDTs and ACTs for them to sell at a subsidised rate. In Tanz3, government providers were paid a supplement to participate in the study. Additional unintentional aspects of studies, such as regular visits or perceived support from evaluators, may have also helped to improve outcomes ${ }^{\text {Uga3,Tanz2 }} .38$
A

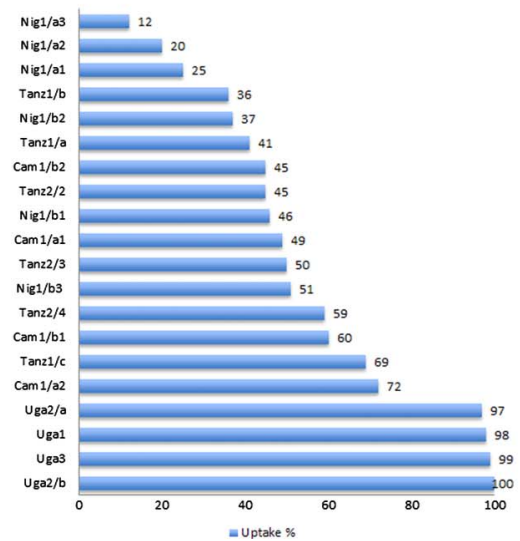

$\mathrm{B}$

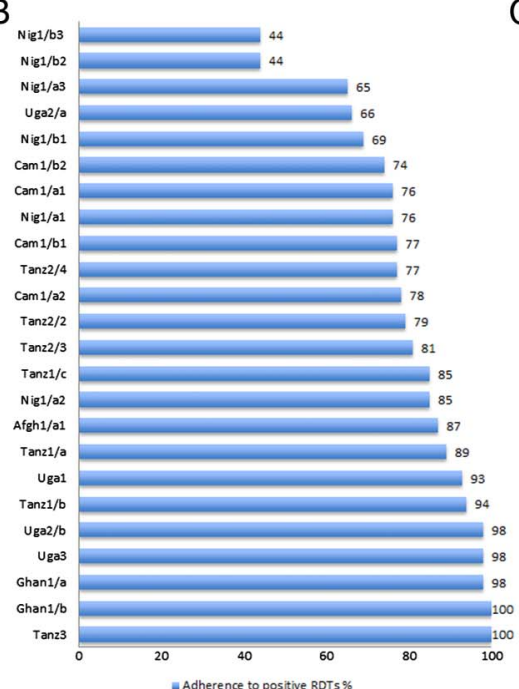

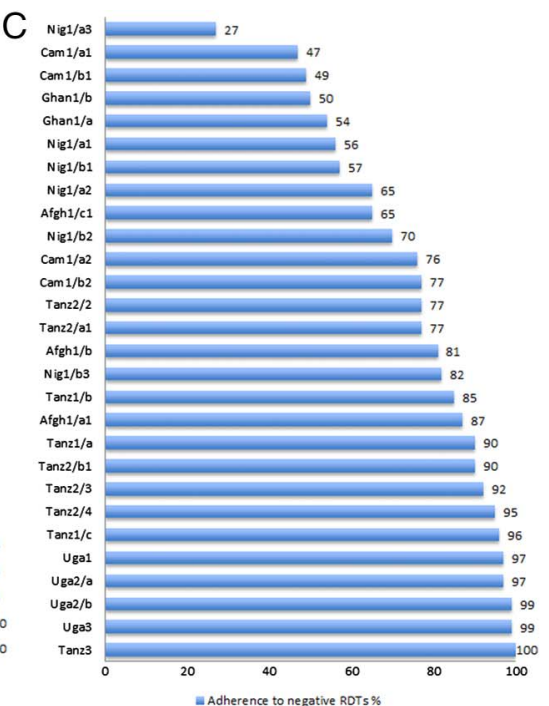

Figure 1 (A) Uptake of malaria rapid diagnostic tests (mRDTs) (\% patients with fever or history of fever who were tested for malaria with an $\mathrm{mRDT}$ ). (B) Adherence to positive mRDT results (\% of patients with a positive mRDT who did receive ACTs). (C) Adherence to negative mRDTs (\% of patients with a negative mRDT results who did NOT receive antimalarials). 
In contrast, where mRDT interventions were not aligned with provider priorities, we saw lower uptake and adherence. For example, in Nig1 in the private sector, providers saw themselves more as vendors than healthcare practitioners. Here, there were anecdotal reports that they were particularly concerned about losing money from sales if mRDT results were negative and wondered whether the public would consider them legitimate to test. This was the case in spite of the free provision of mRDTs to providers by the study team. When providers viewed the intervention as extra unpaid work (eg, conducting tests or recording test results), this affected their motivation. In Uga3, some drug shops declined to participate in the trial for this reason and in Uga1, some health facilities hesitated to continue participating when they felt the work was too much without remuneration. Here, a misalignment between the providers' priorities and the intentions of the intervention led to a lack of motivation for providers to perform in line with guidelines.

\section{Familiarity with testing}

In most cases, there was little prior experience of malaria testing, either using mRDT or microscopy. Although patients were generally keen to be tested for malaria, it was not typically part of providers' routine habits to test. In cases where testing had become part of the established process of care, mRDT uptake tended to be higher. For example, in Tanzl/c, mRDTs had already been scaled up in other districts in recent years, and at baseline there was substantial microscopy testing, unlike the other two cases in this study where uptake was lower Tanz1/a,Tanz1/b. Wide-scale public awareness of testing may have facilitated uptake, for example, in Cameroon, where mass communication campaigns coincided with the study ${ }^{\mathrm{Cam} 1}$, which saw an increase in malaria testing in all study arms from baseline. ${ }^{23}$ Some interventions incorporated local community sensitisation activities to increase familiarity ${ }^{\mathrm{Uga} 2, \mathrm{Uga} 3, \operatorname{Tanz} 2 / 4, \mathrm{Nig} 1 / 3}$, although this appeared insufficient on its own to ensure high uptake.

\section{Adequate human resources and supplies}

Where staff workload was high, or patient numbers exceeded capacity, particularly in small facilities with only one staff member, mRDTs were not always used $^{\text {Uga1,Tanz2/1 }}$.

There were adequate stocks of mRDTs in facilities in most studies, in several cases due to study provision of additional supplies to avert stockouts. However, stockouts

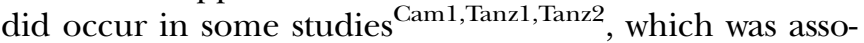
ciated with lower uptake to some extent. Nevertheless, even when mRDTs were available, they were not always used, suggesting other factors were also influential.

\section{Cost of mRDTs to patients}

In most studies, mRDTs were provided free to patients. In those cases where providers were permitted to charge patients for mRDTs, higher prices may have affected their uptake. For example in Nig1, where mRDT uptake was among the lowest observed, patients were charged more than the recommended price on average, particularly in the private sector.

\section{Factors affecting adherence to positive mRDT results}

ACTs were not consistently prescribed to patients with positive mRDT results (see figure 1B). Given the expectation for antimalarial overuse based on previous data, this finding was not anticipated and reasons for low adherence to positive results were therefore not explicitly explored during the studies. However, some explanatory factors driving this outcome did emerge, in addition to the motivation to perform well in the intervention (discussed above). These were the stability of ACT supplies and local preferences for different types of antimalarial.

\section{Stability of ACT supplies}

Stockouts of ACTs were associated with variation in adherence to positive mRDT results; however, this could not explain all the variation. In some cases, ACT use was relatively low despite no or few stockouts, whereas in others, use was high despite stockouts occurring. It may be that provider confidence in the stability of ACT supplies also influenced the use and rationing of ACTs, even when ACTs were available. For example, in Tanz2, lower rates of adherence to positive mRDTs were observed in the case where stockouts were most frequent $^{\text {Tanz2/4 }}$, even after periods of stockouts were excluded from the analysis.

\section{Pre-existing antimalarial preferences}

Information on pre-existing antimalarial preferences was gathered from baseline and preintervention surveys, ${ }^{32} 49$ interview transcripts ${ }^{\text {Tanz1 }}$ and unpublished reports, ${ }^{54}$ although no data were available for five studies ${ }^{\text {Afgh1, }}$ Ghan1,Tanz3,Uga1,Uga2. The data suggest an association between the use of ACTs for positive mRDTs and baseline preferences for, or use of, ACTs rather than other antimalarials. For example, in Nig1, where ACT use was generally low, prior to the intervention, other antimalarials were asked for by patients, prescribed and purchased more commonly than ACTs. ${ }^{34}$ In contrast, in Tanz1, where adherence to RDT positive results was higher, according to stakeholder interviews, ACTs were patients' preferred antimalarial. This may have been due to greater exposure to community sensitisation around $\mathrm{ACTs}^{55}$ or cultural norms around provider authority such that patients felt more inclined to change their preferences in the light of providers' guidance than was the case in Nigeria. An alternative explanation relates to the different roles of the public sector in these countries and therefore, the different influence that the choice of official first-line medicines has on preferences. For example, in Tanzania, public facilities are much more widely used that they are in Nigeria, so people will have 
become used to the idea of ACTs. In Nigeria, the public sector is a more limited provider, so making a drug officially first line may have much less effect on preferences.

\section{Factors affecting adherence to negative mRDT results}

There was also wide variation in the proportion of patients prescribed or given antimalarials in spite of negative mRDT results (see figure $1 \mathrm{C}$ ). In addition to being motivated to perform well in the intervention (discussed above), the analysis suggests adherence to negative mRDTs was also driven in part by the extent to which mRDTs fitted-or were helped by intervention activities to fit-into the existing landscape of care (existing diagnostic and consultation practices). This included providers' perceptions of the role of mRDTs in the diagnostic process and possibilities for alternative diagnoses and treatment. In addition, the analysis suggests that adherence was affected by the extent to which the interventions attempted to control clinical practice.

Malaria tests were usually the only diagnostics available in study facilities. In most cases, test-based malaria diagnosis required a substantial shift from reliance on clinical judgement. In a minority of cases, this shift had already begun before the evaluation started, for example, in Tanzania and Zanzibar where mRDT introductions had begun nationally ${ }^{\text {Tanz1,Tanz3, }}$, or where malaria testing using microscopy was established ${ }^{\text {Afgh1/a,Afgh1/b,Tanz1/c }}$. Here, mRDTs appeared to fit into the landscape of care more easily and adherence to negative mRDT results was higher. Where testing was new and did not fit into the landscape of care so well, even if mRDT use was attractive, adhering to negative results appeared more difficult Afgh1/c,Cam1,Ghan1,Nig1.

Two factors appeared to facilitate integration of mRDTs into the landscape of care: providers' perceptions of the role of mRDTs in the diagnostic process and whether alternative management of illnesses, not involving antimalarials, was possible for those with negative mRDT diagnoses.

\section{Perceived role of mRDTs in diagnostic process}

Two main factors influenced providers' perceptions of the role of mRDTs within the process of malaria diagnosis: how well mRDTs fitted with the dynamic of consultations and whether the mRDT results matched their expectations.

In some cases, providers saw mRDTs as central to the diagnostic process. For example, community health volunteers in Uga2, whose adherence was very high, described the mRDTs as working as 'a judge', and drug shop vendors in Uga3 saw taking blood as crucial to their enhanced role. Conversely, some providers felt clinical judgement should play a more important role in making a diagnosis than mRDTs. Qualitative data suggested that where mRDTs challenged clinicians' expertise and disrupted traditional consultation practices, this led to lower adherence to negative results Afgh1,Ghan1,Tanz2/1. By questioning the test's accuracy, providers were able to reassert their authority and manage the consultation as usual. ${ }^{18} 36$
Some interventions aimed to help mRDTs 'fit' with the dynamics of consultations. For example, training included role-play activities or reflections about how mRDTs would work in practice Cam1/2,Ugal,Uga3, $^{\text {, }}$ experimentation $^{\text {Tanz2/3. Tanz2/4 }}$ and reflection facilitated by multiple training and feedback sessions with

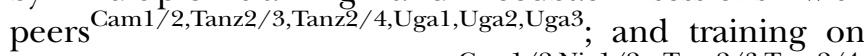

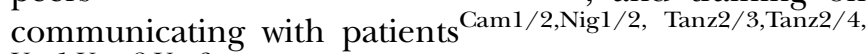
Uga1,Uga 2,Uga3. Providers reported positive impressions of the training's impact on their interactions with patients including the importance of talking to patients and explaining the need for mRDTs or the meaning of their results Ghan1,Tanz2/1,Tanz2/3,Tanz1/a,Uga2.

In some cases, mRDT results did not match expectations; typically, fewer mRDTs were positive than had been expected, particularly when the tests were first

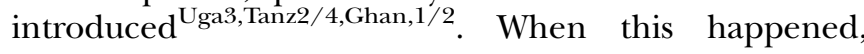
providers placed less emphasis on mRDTs in the diagnostic process, preferring to rely more heavily on clinical judgement. For example, in Cam1/a1, mRDT positivity rates were just $9 \%$, despite the local perception that malaria prevalence was high in that area. Several interviewees from different cases explained that it was hard to trust mRDTs when so many results were negative $^{\text {Ghan1/b,Nig1,Tanz1/b,Tanz2/4,Uga3 }}$, or that they only trusted them once they had seen some positive mRDT results Uga2,Tanz2/4. Providers described a fear of missing malaria diagnoses, particularly when the frequency of positive results was lower than expected, and this was associated with lower adherence ${ }^{\text {Ghan1/1,Ghan1/2,Tanz1/b }}$. In contrast, providers in Tanz3, where adherence to negative mRDTs was high, appeared less concerned about malaria, recognising that prevalence had declined. Some interventions explicitly aimed to raise awareness of current malaria epidemiology during training ${ }^{\text {Tanz2/3, }}$ Tanz2/4,Ugal in order to (re)set expectations of mRDT positivity rates; this was also associated with higher adherence to negative results.

In several cases, providers reported that their trust in mRDTs grew over time ${ }^{\text {Tanz3, Tanz2/2, Tanz2/3, Uga3 }}$. Some described deliberate 'experimentation' to build trust in results, either by testing with microscopy as well as mRDTs $^{\text {Afgh } 1}$ or by seeing whether mRDT-negative patients recovered without antimalarials ${ }^{\text {Ghan1,Uga2 }}$. Indeed in one study, this was explicitly encouraged ${ }^{\text {Tanz2/3, }}$ Tanz2/4. Conversely, some providers' accounts showed mistrust of mRDTs was reinforced by experiences of seeing patients, or indeed themselves, recover when taking antimalarials in spite of a negative mRDT result $\mathrm{Uga}^{\mathrm{Uga} / \mathrm{b}, \mathrm{Ghan} 1 / \mathrm{a}}$. Patient follow-up was considered another useful means of building trust ${ }^{\mathrm{Uga} 2,}$ Ghan1/b. Two interventions aimed to increase the perceived role of mRDTs by providing information about mRDTs' sensitivity and specificity ${ }^{\text {Tanz1,Tanz2/3,Tanz2/4 } 36}$

Alternative treatments for non-malarial fever patients

Interventions offered different options for dealing with mRDT-negative patients (as mentioned above, data on 
the use of alternative treatments are presented in a separate paper). It appeared that expectations and options for alternative management of negative cases-in terms of providers' role, knowledge of case management and availability of other medicines-were important in antimalarial prescribing to mRDT-negative patients. In the public facility interventions where detailed guidance was given to aid alternative diagnoses ${ }^{\text {Ugal,Tanz2,Tanz3 }}$, adherence was higher than in public facilities where no substantial guidance was provided ${ }^{\text {Ghan1,Afgh1 }}$ or where it was recommended that providers only offer antipyretics to mRDT-negative patients ${ }^{\mathrm{Nig} 1 / 2, \mathrm{Nig1} / 3}$. At the community level, where volunteer providers were not expected (or permitted) to provide medicines beyond antimalarials ${ }^{\mathrm{Uga} 2}$, adherence to negative results was high. In private shops in Uganda, where no training on nonmalarial febrile illness management was provided, adherence to mRDT-negative results was still high in terms of ACT prescription, although here mRDT-negative patients ended up being sold other medicines ${ }^{\text {Uga3 }}$.

\section{Directive intervention approach}

Some interventions were more directive about provider practices, particularly regarding the use of unambiguous guidance and supervision or surveillance.

Adherence was typically higher if interventions instructed that no antimalarial should be given to those with negative mRDT results Ugal,Uga2,Uga3,Tanz3. In contrast, adherence was lower when an intervention allowed exceptions for when antimalarials could be given in spite of a negative result, for example, if a febrile patient was under 5 years and had travelled a long distance to seek care Afgh1,Tanz2/2,Cam1. $^{\text {Afar }}$

The highest adherence was observed among providers who had been closely supervised-either for an intense period after training Uga2,Uga3 or throughout the evaluation period ${ }^{\operatorname{Tanz} 3}$. Providers receiving feedback by text message experienced these as a form of surveillance, and reported responding by feeling they should follow guidelines even if their clinical judgement was at odds with this ${ }^{\operatorname{Tanz} 2 / 3, \operatorname{Tanz} 2 / 4}$.

\section{DISCUSSION}

This analysis addresses the persisting gap in knowledge around how to change prescribing practices. This is a key question in this time of international concern over resistance to antimicrobial medicines, with the imperative to optimise medicine use agreed on by United Nations signatories. ${ }^{56}$ By analysing indepth data from 10 co-designed intervention studies from the ACT Consortium, we identify factors affecting the uptake of mRDTs and adherence to test results in different contexts. The varied findings suggest that to improve prescribing through mRDTs, interventions must go beyond basic training in mRDT use and must be tailored to the needs of providers in particular contexts. Uptake and adherence were highest where providers were motivated by the intervention and the tests fitted with the landscape of care. Intervention characteristics that aligned mRDTs with provider priorities included interactive training that addressed how to manage test-negative patients in practice, including clinical and interpersonal aspects of care. Where malaria endemicity is overestimated locally, experimentation and feedback on frequent test-negative cases was important. A directive approach supported by feedback or supervisory instruction can yield high adherence to guidelines but may affect patient-centred care. The results suggest that as mRDTs become established, the intensity of supporting interventions required is likely to reduce.

A strength of this analysis was its use of rich data sources which enabled a more indepth and comprehensive analysis. Although additional insights may have emerged from inclusion of a wider set of studies, synthesising findings from published healthcare interventions is often challenging, with diverse and poorly described interventions, contexts and methods. ${ }^{58} 59$ Nevertheless, our analysis was limited by the fact that not all included studies were able to provide information on all characteristics of interest, while for other characteristics (eg, year and duration), there was too much variation to identify any patterns. While study samples were generally sizeable, in some cases where testing rates and/or malaria prevalence were low, the denominator for adherence outcomes was small. With one exception, where a government mRDT policy was evaluated ${ }^{\text {Tanz1 }}$, all of the evaluated interventions in this analysis were instigated by the study teams. As such, there may be aspects of the interventions, such as RDT supply sources and costs to providers, which may not apply at scale.

Previous studies have identified capacity issues as important in mRDT implementation, such as staffing levels or overworked staff, ${ }^{9}{ }^{12} 60-64$ mRDT or ACT supplies, ${ }^{9} 12$ 61-65 and providers' confidence in mRDT results. ${ }^{12}{ }^{61-66}$ Our synthesis shows that beyond these issues, the introduction of the tests had to make sense in context. Some interventions in our analysis additionally included a more directive approach. While these interventions did achieve the highest rates of adherence to negative results, the consequences of restricting the autonomy of clinicians in favour of standardised guidelines need to be weighed up against the need for clinicians to consider individual patients on a case-by-case basis. ${ }^{67}$ Our finding, that settings where testing was more familiar used mRDTs more appropriately, echoes observations from country-level roll-out of mRDTs, ${ }^{6869}$ and suggests that the interventions required will change over time. Our finding, that basic training alone is insufficient to ensure use of the tests as intended, aligns with findings from studies of interventions aiming to change clinical practice in general. ${ }^{40}$

Prior to introducing mRDTs, initial assessments should be carried out to understand providers' priorities and capacities, as well as how easily tests might integrate into landscapes of care. Although our analysis suggests that a process of tailoring is required to formulate the 
intervention to best fit each context, certain broad intervention features are likely to be applicable across settings (see box 1). As these recommendations arise directly from the data available in our studies, they are not exhaustive.

These findings can inform broader antimicrobial stewardship efforts. Malaria is the first disease for which interventions have been systematically evaluated in order to understand how to change routine prescribing through rapid diagnostics. The lessons learnt in attempting to shift from presumptive to test-directed treatment are relevant for interventions beyond malaria. The intervention and contextual characteristics identified here highlight that apparently simple technological solutions can require complex supporting apparatus when implemented in real life. ${ }^{71}$ However, these findings suggest that as mRDTs become established, the intensity of supporting interventions required is likely to reduce. Further research could explore whether an initial investment in mRDTs could establish patterns of care that

\section{Box 1 Examples of recommended intervention features}

\section{Planning}

Recognise and address providers' priorities

Staffing

Ensure sufficient staff numbers for increased workload

Training

- Offer longer, more detailed training, incorporating interactive activities

- Include training on communicating with patients

- Address process of change to test-based care:

- plan a series of interactive training and/or supervision sessions

- incorporate role-play activities which address local challenges

- use reflective activities

- Build trust in mRDTs by including:

- discussion of data on changes in malaria prevalence in the area

- discussion of sensitivity and specificity of mRDTs

- encouragement to cross-check these data with experience of tests in practice

\section{Guidance}

- Provide detailed guidance and resources for acceptable case management for mRDT-negative patients

- Consider how directive mRDT guidance should be, balancing clarity with the need for clinician judgement to make exceptions (eg, if patients have travelled far, with limited means of transportation to return if their condition worsens)

Medical supplies

- Ensure providers can be confident in supplies of mRDTs and ACTs

- Keep costs to patients low

Community/patient sensitisation

- Conduct patient-oriented sensitisation activities

- where familiarity with testing is low, where frequent falsepositive microscopy has overestimated prevalence, or if ACTs are not the most common antimalarial used or demanded by patients allow for other diagnostic tests to be introduced more easily in the future.

\section{Conclusion}

This analysis shows that uptake and adherence to mRDTs can be high, but this requires either existing contexts where integrating the tests into practice already makes sense, or tailored interventions to encourage this. Basic training and supplies are essential but insufficient to maximise the potential of mRDTs in contexts where they do not fit well with the landscape of care. Apparently simple technological solutions such as mRDTs can require complex supporting interventions that take account of how they will be interpreted and used.

\section{Author affiliations}

${ }^{1}$ Department of Global Health and Development, London School of Hygiene and Tropical Medicine, London, UK

${ }^{2}$ Department of Infectious Disease Epidemiology, London School of Hygiene and Tropical Medicine, London, UK

${ }^{3}$ Epidemiology Unit, Ensign College of Public Health, Kpong, Ghana

${ }^{4}$ Department of Family Health Care Nursing, and Global Health Science,

University of California, Berkeley, California, USA

${ }^{5}$ Department of Microbiology, Tumour and Cell Biology, Karolinska Institute, Stockholm, Sweden

${ }^{6}$ Disease Control Department, London School of Hygiene and Tropical Medicine, London, UK

${ }^{7}$ Clinical Research Department, London School of Hygiene and Tropical Medicine, London, UK

${ }^{8}$ Department of Infectious Diseases, Sahlgrenska Academy, University of Gothenburg, Goteborg, Sweden

${ }^{9}$ Department of Paediatrics, Sahlgrenska Academy, University of Gothenburg, Goteborg, Sweden

${ }^{10}$ Department of Microbiology, Tumour and Cell Biology, Karolinska Institutet, Stockholm, Sweden

${ }^{11}$ Faculty of Health and Medical Sciences, Centre for Medical Parasitology, University of Copenhagen, Copenhagen, Denmark

${ }^{12}$ Department of Women's and Children's Health, Uppsala University, Uppsala, Sweden

${ }^{13}$ Laboratory for Public Health Research Biotechnologies, The Biotechnology Center, University of Yaoundé, Yaoundé, Cameroon

${ }^{14}$ School of Public Health- Makerere University and Commissioner Health

Services, Ministry of Health, Uganda

${ }^{15}$ Department of Pharmacology and Therapeutics, University of Nigeria EnuguCampus, Nigeria

${ }^{16}$ Centers for Disease Control and Prevention (CDC), USA

${ }^{17}$ Department of Medicine, Kungälv Hospital, Sweden

${ }^{18}$ Centre for Medical Parasitology, University of Copenhagen and Copenhagen University Hospital Rigshospitalet, Denmark

${ }^{19}$ Department of Infectious Disease Epidemiology, Statens Serum Institut,

Denmark

${ }^{20}$ School of Public Health and Community Medicine, Australia

Correction notice This article has been corrected since it first published. Figure 1 has been replaced with the correct version.

Acknowledgements This research was funded by the ACT Consortium through a grant from the Bill and Melinda Gates Foundation to the London School of Hygiene and Tropical Medicine. The authors gratefully acknowledge the contribution of colleagues involved in each of the studies included in the analysis, in particular those who collected data, conducted analysis or contributed to the concept of this analysis: Bonnie Cundill, Catherine Maiteki, Clarence Mkoba, Evelyn Ansah, Ismail Mayan, Lindsay Mangham Jefferies, Mark Rowland, Mwinyi Msellem, Patrick Kachur, Rebecca Thomson, Renata Mandike, Richard Ndyomugyenyi, Seth Owusu-Agyei, Shunmay Yeung, Toby Leslie, Jo Reynolds, Hugh Reyburn, David Lalloo and David Schellenberg. The authors would also like to thank all other participants in the included studies: 
the patients and their guardians, providers, data collectors and other study team members. LSV is an employee of the WHO and DRA is an employee of the Centres for Disease Control and Prevention.

Disclaimer The views expressed in this article are the views of the authors and may not necessarily reflect the views of the WHO or CDC.

Contributors HEDB and CIRC designed the study. HEDB conducted the analysis and drafted the paper; $\mathrm{CIRC}$ contributed to analysis and drafting. $\mathrm{BL}$, $\mathrm{FB}, \mathrm{KB}, \mathrm{AB}, \mathrm{KBr}, \mathrm{SC}, \mathrm{DDL}, \mathrm{KE}, \mathrm{CG}, \mathrm{HH}, \mathrm{SL}, \mathrm{PM}, \mathrm{AM}, \mathrm{WM}, \mathrm{AMb}, 00$, DRA, $\mathrm{DS}, \mathrm{SS}$ and LSV contributed to data collection. All authors contributed to study design, analysis and the final write-up and approved the manuscript.

Funding This analysis, as well as the projects it included, was funded by the Bill and Melinda Gates Foundation, grant number 39640.

Competing interests None declared.

Patient consent No.

Ethics approval ZAMREC, Zanzibar; Ghana Health Service Ethical Review Committee; UNCST; MU SOMREC; Ministry of Health and National Institute of for Medical Research, Tanzania; Ministry of Health Institutional Review Board Afghanistan; University Committee on Medical and Scientific Research Ethics, Nigeria; National Ethics Committee, Cameroon; Makerere University IRB; Uganda National Council for Science and Technology; LSHTM; University of California San Francisco Committee on Human Research; CDC; IHI, NIMR.

Provenance and peer review Not commissioned; externally peer reviewed.

Data sharing statement Data from the studies included in this analysis can be found at the ACTc repository: https://actc.Ishtm.ac.uk. This includes outcome data, description of intervention and data collection tools.

Open Access This is an Open Access article distributed in accordance with the terms of the Creative Commons Attribution (CC BY 4.0) license, which permits others to distribute, remix, adapt and build upon this work, for commercial use, provided the original work is properly cited. See: http:// creativecommons.org/licenses/by/4.0/

\section{REFERENCES}

1. World Health Organization (WHO). New Perspectives: Malaria Diagnosis. Report of a joint WHO/USAID Informal consultation, 2527 October 1999. Geneva, Switzerland: World Health Organization (WHO), 2000

2. World Health Organization (WHO). T3. Test. Treat. Track. Scaling up diagnostic testing, treatment and surveillance for malaria. Geneva: Global Malaria Programme, 2012 http://www.who.int/malaria/ publications/atoz/t3_brochure/en/

3. World Health Organization (WHO). The use of malaria rapid diagnostic tests. 2nd edn. Geneva, Switzerland: WHO, 2006.

4. Bell D, Perkins MD. Making malaria testing relevant: beyond test purchase. Trans R Soc Trop Med Hyg 2008;102:1064-6.

5. Drakeley $\mathrm{C}$, Reyburn $\mathrm{H}$. Out with the old, in with the new: the utility of rapid diagnostic tests for malaria diagnosis in Africa. Trans $R$ Soc Trop Med Hyg 2009;103:333-7.

6. World Health Organization (WHO). Guidelines for the treatment of malaria. 2nd edn. Geneva: WHO, 2010.

7. Mendelson M, Rottingen JA, Gopinathan U, et al. Maximising access to achieve appropriate human antimicrobial use in low-income and middle-income countries. Lancet 2016;387:188-98.

8. Odaga J, Lokong JA, Donegan S, et al. Rapid diagnostic tests versus clinical diagnosis for managing people with fear in malaria endemic settings. Cochrane Database Syst Rev 2014;(4):CD008998.

9. Rao VB, Schellenberg D, Ghani AC. Overcoming health systems barriers to successful malaria treatment. Trends Parasitol 2013;29:164-80.

10. Johansson EW, Gething PW, Hildenwall $\mathrm{H}$, et al. Diagnostic testing of pediatric fevers: meta-analysis of 13 national surveys assessing influences of malaria endemicity and source of care on test uptake for febrile children under five years. PLOS ONE 2014;9:e95483.

11. Johansson EW, Gething PW, Hildenwall $\mathrm{H}$, et al. Effect of diagnostic testing on medicines used by febrile children less than five years in 12 malaria-endemic African countries: a mixed-methods study. Malar J 2015;14:194.

12. Ochodo E, Garner $P$, Sinclair D. Achieving universal testing for malaria. BMJ 2016;352:i107.

13. http://www.actconsortium.org/
14. Pope C, Mays J, Popay J. Synthesizing qualitative and quantitative health evidence: a guide to methods. Berkshire, England: McGraw Hill/Open University Press, 2007.

15. Yin RK. Case study research: design and methods. 4th edn. London: SAGE Publications, 2009.

16. Hopkins $\mathrm{H}$, Bruxvoort KJ, Cairnes ME, et al. The impact of introducing malaria rapid diagnostic tests on antibiotic prescribing: a nine-site analysis in public and private health care settings. BMJ 2017, in press.

17. Crowe S, Cresswell K, Robertson A, et al. The case study approach. BMC Med Res Methodol 2011;11:100.

18. Reynolds J, Wood M, Mikhail A, et al. Malaria "diagnosis" and diagnostics in Afghanistan. Qual Health Res 2013;23:579-91.

19. Leslie T, Mikhail A, Mayan I, et al. Overdiagnosis and mistreatment of malaria among febrile patients at primary healthcare level in Afghanistan: observational study. BMJ 2012;345: e4389.

20. Leslie T, Mikhail A, Mayan I, et al. Rapid diagnostic tests to improve treatment of malaria and other febrile illnesses: patient randomised effectiveness trial in primary care clinics in Afghanistan. BMJ 2014;348:g3730.

21. Chandler $\mathrm{Cl}$, Mangham L, Njei AN, et al. 'As a clinician, you are not managing lab results, you are managing the patient': how the enactment of malaria at health facilities in Cameroon compares with new WHO guidelines for the use of malaria tests. Soc Sci Med 2012;74:1528-35

22. Mangham LJ, Cundill B, Achonduh OA, et al. Malaria prevalence and treatment of febrile patients at health facilities and medicine retailers in Cameroon. Trop Med Int Health 2012;17:330-42.

23. Mbacham WF, Mangham-Jefferies L, Cundill B, et al. Basic or enhanced clinician training to improve adherence to malaria treatment guidelines: a cluster-randomised trial in two areas of Cameroon. Lancet Glob Health 2014;2:e346-58.

24. Wiseman V, Mangham LJ, Cundill B, et al. A cost-effectiveness analysis of provider interventions to improve health worker practice in providing treatment for uncomplicated malaria in Cameroon: a study protocol for a randomized controlled trial. Trials 2012;13:4.

25. Achonduh OA, Mbacham WF, Mangham-Jefferies L, et al. Designing and implementing interventions to change clinicians' practice in the management of uncomplicated malaria: lessons from Cameroon. Malar J 2014;13:204

26. Mangham-Jefferies L, Wiseman V, Achonduh OA, et al. Economic evaluation of a cluster randomized trial of interventions to improve health workers' practice in diagnosing and treating uncomplicated malaria in Cameroon. Value Health 2014;17:783-91.

27. Mangham-Jefferies L, Hanson K, Mbacham W, et al. What determines providers' stated preference for the treatment of uncomplicated malaria? Soc Sci Med 2014;104:98-106.

28. Ansah EK, Narh-Bana S, Epokor M, et al. Rapid testing for malaria in settings where microscopy is available and peripheral clinics where only presumptive treatment is available: a randomised controlled trial in Ghana. BMJ 2010;340:c930.

29. Ansah EK, Reynolds J, Akanpigbiam S, et al. 'Even if the test result is negative, they should be able to tell us what is wrong with us': a qualitative study of patient expectations of rapid diagnostic tests for malaria. Malar J 2013;12:258.

30. Chandler $\mathrm{Cl}$, Whitty $\mathrm{CJ}$, Ansah EK. How can malaria rapid diagnostic tests achieve their potential? A qualitative study of a tria at health facilities in Ghana. Malaria J 2010:9:95.

31. Ezeoke OP, Ezumah NN, Chandler CC, et al. Exploring health providers' and community perceptions and experiences with malaria tests in South-East Nigeria: a critical step towards appropriate treatment. Malar J 2012;11:368.

32. Mangham-Jefferies L, Hanson K, Mbacham W, et al. Mind the gap: knowledge and practice of providers treating uncomplicated malaria at public and mission health facilities, pharmacies and drug stores in Cameroon and Nigeria. Health Policy Plan 2015;30:1129-41.

33. Wiseman V, Ogochukwu E, Emmanuel N, et al. A cost-effectiveness analysis of provider and community interventions to improve the treatment of uncomplicated malaria in Nigeria: study protocol for a randomized controlled trial. Trials 2012;13:81.

34. Mangham LJ, Cundill B, Ezeoke O, et al. Treatment of uncomplicated malaria at public health facilities and medicine retailers in south-eastern Nigeria. Malar J 2011;10:155.

35. Bruxvoort K, Kalolella A, Nchimbi H, et al. Getting antimalarials on target: impact of national roll-out of malaria rapid diagnostic tests on health facility treatment in three regions of Tanzania. Trop Med Int Health 2013;18:1269-82.

36. Chandler Cl, Meta J, Ponzo C, et al. The development of effective behaviour change interventions to support the use of malaria rapid diagnostic tests by Tanzanian clinicians. Implement Sci 2014;9:83 
37. Cundill B, Mbakilwa $\mathrm{H}$, Chandler $\mathrm{Cl}$, et al. Prescriber and patient-oriented behavioural interventions to improve use of malaria rapid diagnostic tests in Tanzania: facility-based cluster randomised trial. BMC Med 2015;13:118.

38. Leurent $\mathrm{B}$, Reyburn $\mathrm{H}$, Muro $\mathrm{F}$, et al. Monitoring patient care through health facility exit interviews: an assessment of the Hawthorne effect in a trial of adherence to malaria treatment guidelines in Tanzania. BMC Infect Dis 2016;16:59.

39. Baltzell K, Elfving K, Shakely D, et al. Febrile illness management in children under five years of age: a qualitative pilot study on primary health care workers' practices in Zanzibar. Malar J 2013;12:37.

40. Shakely D, Elfving K, Aydin-Schmidt B, et al. The usefulness of rapid diagnostic tests in the new context of low malaria transmission in Zanzibar. PLOS ONE 2013;8:e72912.

41. Chandler Cl, DiLiberto D, Nayiga S, et al. The PROCESS study: a protocol to evaluate the implementation, mechanisms of effect and context of an intervention to enhance public health centres in Tororo, Uganda. Implement Sci 2013;8:113.

42. Staedke SG. Evaluating the impact of a public health centre intervention on management of malaria and health outcomes of children in Uganda -results from the PRIME \& PROCESS studies. Policy Brief 2014. http://www.actconsortium.org/data/files/resources/109/PRIME-andPROCESS-policy-brief-English.pdf (accessed 7 Jun 2016)

43. Staedke SG, Chandler CI, DiLiberto D, et al. The PRIME trial protocol: evaluating the impact of an intervention implemented in public health centres on management of malaria and health outcomes of children using a cluster-randomised design in Tororo, Uganda. Implement Sci 2013;8:114.

44. DiLiberto DD, Staedke SG, Nankya F, et al. Behind the scenes of the PRIME intervention: designing a complex intervention to improve malaria care at public health centres in Uganda. Glob Health Action 2015;8:29067.

45. Lal S, Ndyomugyenyi R, Alexander ND, et al. Health facility utilisation changes during the introduction of community case management of malaria in South Western Uganda: an interrupted time series approach. PLOS ONE 2015;10:e0137448.

46. Mbonye AK, Ndyomugyenyi R, Turinde A, et al. The feasibility of introducing rapid diagnostic tests for malaria in drug shops in Uganda. Malar J 2010;9:367.

47. Chandler $\mathrm{Cl}$, Hall-Clifford R, Asaph $\mathrm{T}$, et al. Introducing malaria rapid diagnostic tests at registered drug shops in Uganda: limitations of diagnostic testing in the reality of diagnosis. Soc Sci Med 2011;72:937-44

48. Hutchinson E, Chandler C, Clarke S, et al. 'It puts life in us and we feel big': shifts in the local health care system during the introduction of rapid diagnostic tests for malaria into drug shops in Uganda. Crit Public Health 2015;25:48-62.

49. Mbonye AK, Lal S, Cundill B, et al. Treatment of fevers prior to introducing rapid diagnostic tests for malaria in registered drug shops in Uganda. Malar J 2013;12:131.

50. Mbonye AK, Magnussen $\mathrm{P}$, Chandler $\mathrm{Cl}$, et al. Introducing rapid diagnostic tests for malaria into drug shops in Uganda: design and implementation of a cluster randomized trial. Trials 2014;15:303.

51. Mbonye AK, Magnussen P, Lal S, et al. A cluster randomised trial introducing rapid diagnostic tests into registered drug shops in Uganda: impact on appropriate treatment of malaria. PLOS ONE 2015;10:e0129545.

52. Sutcliffe K, Thomas J, Stokes G, et al. Intervention component analysis (ICA): a pragmatic approach for identifying the critical features of complex interventions. BMC Syst Rev 2015;4:f3755.

53. Thomas J, O'Mara-Eves A, Brunton G. Using qualitative comparative analysis (QCA) in systematic reviews of complex interventions: a worked example. Syst Rev 2014;3:67.

54. Meta J, Nasuwa F, Kessy J, et al. TACT Formative Research Analysis Report, 2010.

55. Willey BA, Tougher S, Ye Y, et al. Communicating the AMFm message: exploring the effect of communication and training interventions on private for-profit provider awareness and knowledge related to a multi-country anti-malarial subsidy intervention. Malar $\mathrm{J}$ $2014 ; 13: 46$
56. World Health Organisation. Global Action Plan on Antimicrobial Resistance. Geneva, 2015, http://www.who.int/drugresistance/ global_action_plan/en/

57. United Nations. Draft political declaration of the high-level meeting of the General Assembly on antimicrobial resistance. 2016. http://www. un.org/pga/71/wp-content/uploads/sites/40/2016/09/DGACM GAEAD ESCAB-AMR-Draft-Political-Declaration-1616108E.pd

58. Michie S, Fixsen D, Grimshaw JM, et al. Specifying and reporting complex behaviour change interventions: the need for a scientific method. Implement Sci 2009;4:40.

59. Hoffmann TC, Erueti C, Glasziou PP. Poor description of non-pharmacological interventions: analysis of consecutive sample of randomised trials. BMJ 2013;347: $\{3755$

60. Kyabayinze DJ, Asiimwe C, Nakanjako D, et al. Programme level implementation of malaria rapid diagnostic tests (RDTs) use: outcomes and cost of training health workers at lower level health care facilities in Uganda. BMC Public Health 2012;12:291.

61. Febir LG, Baiden FE, Agula J, et al. Implementation of the integrated management of childhood illness with parasitological diagnosis of malaria in rural Ghana: health worker perceptions. Malar J 2015;14:174.

62. Mubi M, Kakoko D, Ngasala B, et al. Malaria diagnosis and treatment practices following introduction of rapid diagnostic tests in Kibaha District, Coast Region, Tanzania. Malar J 2013;12:293.

63. Bastiaens GJ, Bousema T, Leslie T. Scale-up of malaria rapid diagnostic tests and artemisinin-based combination therapy: challenges and perspectives in sub-Saharan Africa. PLOS Med 2014;11:e1001590.

64. Boadu NY, Amuasi J, Ansong D, et al. Challenges with implementing malaria rapid diagnostic tests at primary care facilities in a Ghanaian district: a qualitative study. Malar $J$ 2016;15:126.

65. Diggle E, Asgary R, Gore-Langton G, et al. Perceptions of malaria and acceptance of rapid diagnostic tests and related treatment practises among community members and health care providers in Greater Garissa, North Eastern Province, Kenya. Malar J 2014; 13:502.

66. Asiimwe C, Kyabayinze DJ, Kyalisiima Z, et al. Early experiences on the feasibility, acceptability, and use of malaria rapid diagnostic tests at peripheral health centres in Uganda-insights into some barriers and facilitators. Implement Sci 2012;7:5

67. Armstrong D. Clinical autonomy, individual and collective: the problem of changing doctors' behaviour. Soc Sci Med 2002;55:1771-7.

68. Faust $\mathrm{C}$, Zelner $\mathrm{J}$, Brasseur $\mathrm{P}$, et al. Assessing drivers of full adoption of test and treat policy for malaria in Senegal. Am J Trop Med Hyg 2015;93:159-67.

69. Zurovac D, Githinji S, Memusi D, et al. Major improvements in the quality of malaria case-management under the "test and treat" policy in Kenya. PLOS ONE 2014;9:e92782.

70. Greenhalgh T, Swinglehurst D. Studying technology use as social practice: the untapped potential of ethnography. BMC Med 2011;9:45.

71. Beisel U, Umlauf R, Hutchinson $\mathrm{E}$, et al. The complexities of simple technologies: re-imagining the role of rapid diagnostic tests in malaria control efforts. Malar J 2016;15:64.

72. Chandler CIR, Webb EL, Maiteki-Sebuguzi C, et al. The impact of an intervention to introduce malaria rapid diagnostic tests on fever case management in a high transmission setting in Uganda: A mixed-methods cluster-randomised trial (PRIME). Plos One. In press.

73. Staedke, SG, Maiteki-Sebuguzi, C, DiLiberto DD. The impact of an intervention to improve malaria care in public health centers on health indicators of children in Tororo, Uganda (PRIME): A clusterrandomised trial. Am J Trop Med Hyg 2016;95:358-67.

74. Ndyomugyenyi R, Magnussen P, Lal S, et al. Appropriate targeting of artemisini-based combination therapy by community health workers using malaria rapid diagnostic tests: findings from randomized trials in two contrasting areas of high and low malaria transmission in south western Uganda. Tropical Medicine and International Health 2016;21:1157-70. 